\title{
MULTI-FREQUENCY AND MULTI-POLARIZATION ANALYSIS OF OIL SLICKS USING TERRASAR-X AND RADARSAT-2 DATA
}

\author{
Suman Singha; Rudolf Ressel; Susanne Lehner \\ Maritime Security Research Center \\ Remote Sensing Technology Institute (IMF), German Aerospace Center (DLR) \\ Heinrich Focke Str. 4, 28199, Bremen, Germany \\ Suman.Singha@dlr.de; Rudolf.Ressel@dlr.de; Susanne.Lehner@dlr.de
}

\begin{abstract}
The use of fully polarimetric SAR data for oil spill detection is relatively new and shows great potential for operational offshore platform monitoring. Greater availability of these kind of SAR data calls for a development of time critical processing chain capable of detecting and distinguishing oil spills from 'look-alikes'. This paper describes the development of an automated Near Real Time (NRT) oil spill detection processing chain based on quad-pol RADARSAT-2 (RS-2) and quad-pol TerraSAR-X (TS-X) images, wherein we use polarimetric features (e.g. Lexicographic and Pauli Based features) to characterize oil spills and look-alikes. Numbers of TS-X and RS-2 images have been acquired over known offshore platforms along with some near coincident (spatially and temporally) acquisition. Ten polarimetric feature parameters were extracted from different types of oil (e.g. crude oil, emulsion etc) and 'look-alike' (e.g. plant oil, met-oceanic phenomenon etc) spots and divided into training and validation dataset seperately for TerraSAR-X RADARSAT-2. Extracted features were then used for training and validation of a pixel based Artificial Neural Network (ANN) classifier. Initial performance estimation was carried out for the proposed methodology in order to evaluate its suitability for NRT operational service. Mutual information contents among extracted features were assessed and feature parameters were ranked according to their ability to discriminate between oil spills and look- alikes. Polarimetric features such as Scattering Diversity and Pauli-based features proved to be more discriminative than other polarimetric features.
\end{abstract}

Index Terms- Oil Spill Detection; Feature Extraction, Feature Ranking; Pol-SAR, TerraSAR-X, RADARSAT-2.

\section{INTRODUCTION}

In last few years, a number of semi-automatic and automatic techniques have been developed in order to differentiate oil

TS-X/TD-X Quad Pol Images were acquired through TanDEM-X Science AO: NTI_POLI6690 (PI: Suman Singha) and RADARSAT-2 images were acquired through ESA Category-1 Project Id. C1F.16331 spill and look alike spots based on single pol SAR data using traditional feature parameters (Geometric, Shape, Texture etc.). On the other hand, numbers of polarimetric features have been proposed recently for the same purpose [1] [2] [3] [4] [5] based on experimental dataset. Although, an integration of traditional feature along with polarimetric features into the oil spill detection methodologies are yet to be established [6]. In order to mitigate this situation a major focus of research in this area is the development of automated algorithms based on polarimetric features to distinguish oil spills from look-alikes complementing traditional features currently in use.

As SAR sensors with Dual (HH-VV) and Quad polarimetric data acquisition capabilities are now operationally available from several L (ALOS PALSAR), C (RADARSAT-2, RISAT) and X (TerraSAR-X, COSMO SkyMed) band SAR sensors and methodologies for polarimetric based oil spill detection systems are evolving rapidly, development of operational near real time processing chain based on SAR polarimetry is highly desirable. Although many previous research based on single polarimetric images manage to produce reasonable classification accuracy, they suffers from significant false positive rate. Recent studies shows unique benefits of polarimetric SAR data to both observe oil slicks and discrimination between oil spill and look-alike spots which is a major challenge for traditional oil spill detection systems based on single polarized SAR images [1]-[5]. Concept for using Polarimetric SAR data for oil slick observation was initially demonstrated by in [2] [3] on $\mathrm{C}$ and $\mathrm{L}$ band multilook complex data. The basic concept was then adapted for X-band in [1] using TerraSAR-X dual pol single look complex data. The polarimetric electromagnetic model which is the basis of this proposed methodology predicts that sea surface governed by Bragg scattering, have high interchannel (co-pol) correlation and in case of non-Bragg scatter the correlation is significantly lower. In this context, [1] demonstrated that standard deviation of Co polarized phase difference for polluted area is significantly higher compared to pollution free background. In [5], evaluation of eight well established and mod- 
ified polarimetric feature have been carried out on C-band RADARSAT-2 fine quad pol data. Proposed methodology uses recently established polarimetric features to characterize dark spots with the help of ANN.

\section{DATASET}

Fully polarimetric TerraSAR-X (TS-X) and RADARSAT-2 (RS-2) images have been used to develop and validate the proposed algorithm. Majority of the images were acquired over Bombay High region, about 160 kilometres off the coast of Mumbai, India. This region host a cluster of offshore oil platforms and in production since 1974. Unlike European waters, there are no observation derived from regular aerial surveillance, therefore the only possible way to monitor this kind of area, is the use satellite SAR imagery. In addiition to dataset set collected over Bombay High region we have also gathered a number of TS-X and RS-2 fully polarimetric images manifesting 'look-alike' spots.

From March 2015 until the October 2015, A total number of 20 TerraSAR-X images and 20 RADARSAT- 2 images were acquired over the study area. The fully polarimetric $X$-band SAR data have been acquired by TerraSAR-X and TanDEM-X constellation during a scientific exploration campaign started in October 2014 and continued until December 2015. One of the main objectives of this campaign is to evaluate quad-polarized X-band high resolution SAR data to develop new technologies and applications. X-band quad pol data were acquired using Dual Receive Antenna (DRA) configuration mode. DRA configuration is achieved by electronically splitting the physical antenna (4.8m in Azimuth) during receive into two parts separated in along track ([7]). Therefore, in order to obtain the full scattering matrix the whole antenna is transmitting one polarisation (toggled pulse by pulse to transmit two polarization) and in reception, the split of antenna enables recording of two polarizations ([8]). The Xband fully polarimetric data which were acquired over the study area is quite unique as currently, only TerraSAR-X and TanDEM-X constellation is able to provide such space-borne observation (only during science phase). On the other hand C-band RADARSAT-2 Quad pol SAR data is available in two different modes, Standard Quad pol and Fine Quad Pol. In both quad polarization modes, a nominal image scene covers an area of approximately $25 \mathrm{~km}$ (Range) $\times 25 \mathrm{~km}$ (Azimuth). These two modes of quad pol data is also available in 'Wide' mode, where a nominal image scene covers an area of approximately $50 \mathrm{~km}$ (Range) $\times 25 \mathrm{~km}$ (Azimuth). In case of TerraSAR-X StripMap quad polarization mode a nominal image scene covers an area of approximately $16 \mathrm{~km}$ (Range) $\times$ $55 \mathrm{~km}$ (Azimuth).

All of the images acquired, were Single-Look Complex (SLC) product with nominal slant range resolution of approximately $1.2 \mathrm{~m}$ in case of TerraSAR-X StripMAP and $5.2 \mathrm{~m}$ in case of RADARSAT-2 Fine Quad (FQ) and Fine Quad Wide
(FQW) which is ideal for monitoring offshore platform clusters like Bombay High. The dataset includes different beams of TerraSAR-X StripMAP mode (e.g. StripFar_006, StripNear_009 etc.) and RADARSAT-2 Fine Quad pol Mode (e.g. FQ10, FQ13, FQ6W etc.) incorporating a broad range of incidence angle, from 20 to 35 degree. Incidence angle range was chosen due to it's low instrument noise floor (NESZ range [$19 \mathrm{~dB} ;-26 \mathrm{~dB}]$ in case of TerraSAR-X and $-36.5 \pm 3 \mathrm{~dB}$ in case of RADARSAT-2) which is a critical parameter in respect to the polarimetric system performance and low enough for dark feature observation even at low wind condition $([9,1])$. The dataset is strongly heterogeneous, contains several oil spill spots, providing fresh and old platform sourced spills and incorporating different wind conditions. All the TS-X and RS-2 images present in the dataset were in the range of desirable wind conditions which is around 3-13 m/s. Moreover, the dataset contains a comprehensive set of look-alikes, including phenomena such as ship wakes and low wind areas. A small number of look-alikes present in the dataset are not from the study area.

Figure 1 shows an example of a platform sourced oil spills along with presence of the offshore platform cluster on a RS-2 (Top left) Fine quad pol wide image acquired on 15th of July 2015 at 01:16 UTC (Descending orbit) and on a TS-X (Lower left) StripMap quadpol image acquired just one minute before the RS-2 acquisition over the same study area. As mentioned earlier some of the look-alike examples were collected outside of the study area as occurrences of look-alike over the study area is very limited. Figure 2 demonstrate manifestations of look-alike phenomenon probably due to biogenic slicks on a TS-X quad-pol image acquired over North Sea.

\section{METHODOLOGY}

First step of the proposed methodology is standard meta-data retrieval, pre-processing and calibration. From each image ten polarimetric features are extracted (Table 1). As mentioned in the introduction, we performed a pixel-based supervised classification using a (artificial) neural network (NN, ANN). Training and validation dataset were extracted from 5 TS-X and 4 RS-2 images for four separate classes, Crude Oil (CO), Emulsion (EM), Look-Alike (LA) and Open Water (OW). It is important to note that the training and validation datasets are mutually exclusive and some of the training and validation dataset (RS-2) were extracted form verified Crude Oil (CO), Emulsion (EM), Look-Alike (LA) spots, previously presented and studied by [5] and [4]. The feature set we used contained all features and additionally their respective local variances, which was computed for each center pixel of a $11 \times 11$ submatrix sliding over the entire feature image. Thus, we extracted for each pixel, mentioned polarimetric features and then ingested the feature vectors into the classifier. The implementation was carried out in the Exelis IDL programming language (Image ingestion, calibration, feature extrac- 

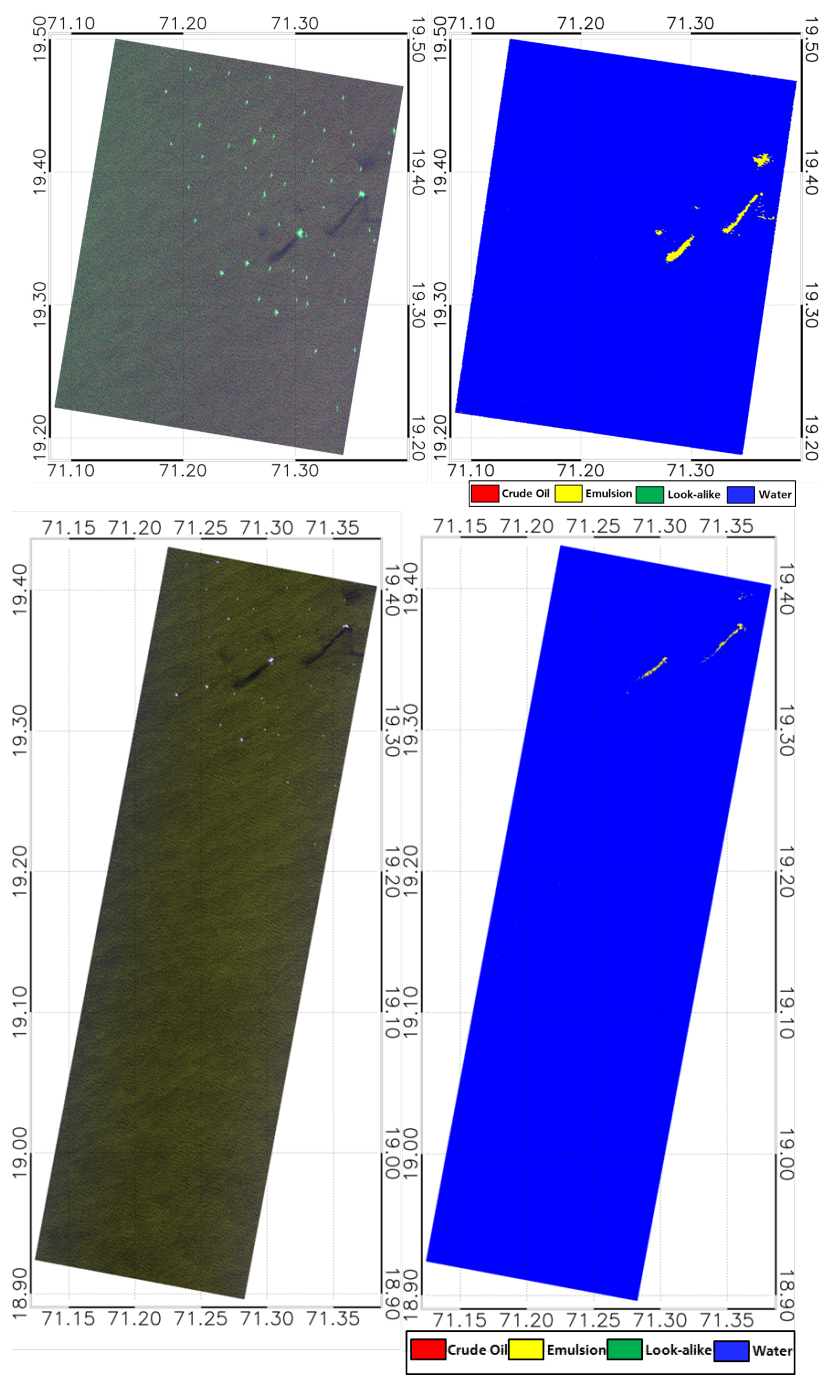

Fig. 1. $\mathrm{C}$ and $\mathrm{X}$ band fully polarimetric SAR imagery acquired over Bombay High and corrosponding classified imagery. Top left: Pauli representation of RADARSAT-2 Fine Quad Pol Wide (FQ4, mid incidence angle $\theta=23.2^{\circ}$, Descending Orbit) acquired on 15th of July 2015 at 01:16 UTC. Top right: Classified RS-2 image using ANN. Lower left: TerraSAR-X Quad Pol (Dual Receive Antenna, StripFar_006, mid incidence angle $\theta=29.3^{\circ}$, Descending Orbit) acquired on 15th of July 2015 at 01:15 UTC. Lower right: Classified TS-X image using ANN.

tion, statistical analysis) and in C (FANN library classifier). The hardware specifications used were: 11 GB RAM, Intel Core i-7 3740 QM, virtual linux OS.

\section{RESULTS AND DISCUSSION}

As discussed in the section 3 we train the ANN with training dataset consist of ten polarimetric feature and there local vari-

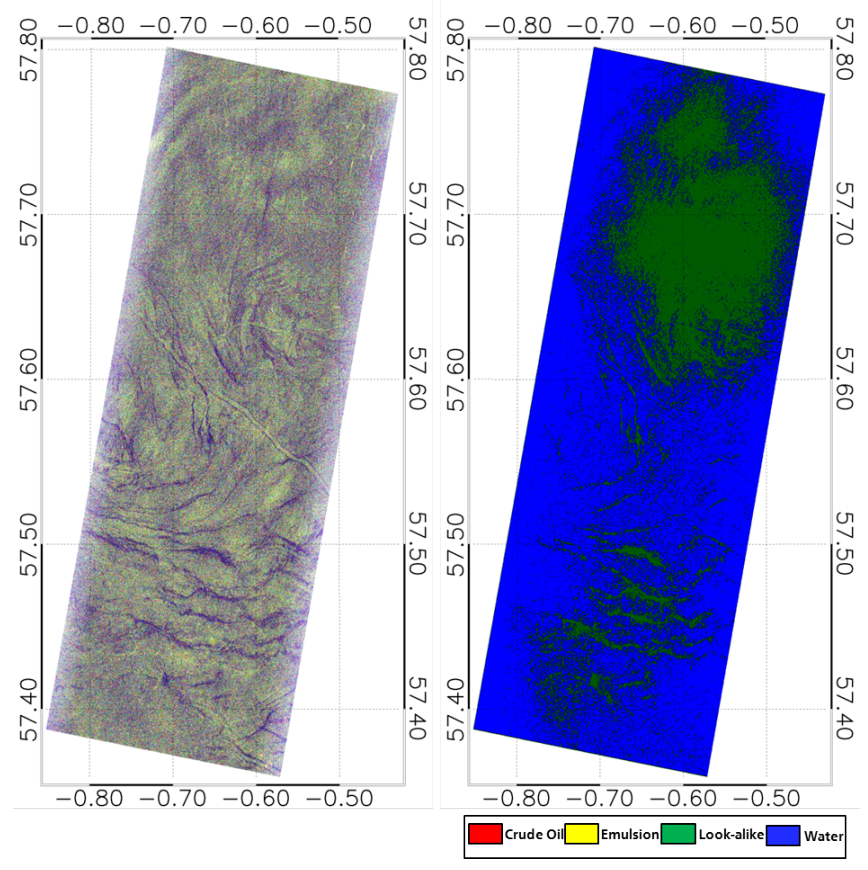

Fig. 2. Left: Manifestation of 'look-alikes' (Algae/Biogenic Slick) on StripMAP image (mid incidence angle: $\theta=38.80^{\circ}$, stripNear_011, Contrast enhanced) acquired on 13th of July 2015 at 06:24 UTC over NorthSea. Right: Classification result using ANN. (C) DLR,2015).

Table 1. List of polarimetric features used in this study

\begin{tabular}{|c|}
\hline Polarimetric features \\
\hline Stddev Co-Pol Phase Difference $\sigma_{\varphi, C O}[1],[3]$ \\
\hline Coherency Coefficient $\rho_{C O}[1]$ \\
\hline Geometric Intensity $\mu[5]$ \\
\hline Real Part Co-Pol Cross Product [3] \\
\hline Entropy $H[5]$ \\
\hline Anisotropy $A[5]$ \\
\hline Alpha angle $\alpha[5]$ \\
\hline Scattering Diversity $\gamma_{C O}[10]$ \\
\hline Surface Scattering Fraction $\gamma_{C O}[10]$ \\
\hline Polarimetric Span $[11]$ \\
\hline
\end{tabular}

ance. After training we tested the performance of the trained ANN using a testing dataset. Table 2 and 3 show the classification accuracy achieved by the trained ANN using testing dataset for RS-2 and TS-X respectively. Noting that both training and testing dataset are mutually exclusive and collected from the different images (i.e. different met-oceanic conditions and incidence angle), our approach can be considered to be consistent in itself and stable in terms of the coping with different met-oceanic conditions as background. Additionally, the proposed methodology is tuned for operational Near Real Time Services with average processing time of 7-8 min for a RS-2 fine quad pol scene and 10-12 min for a TS-X 
StripMap quad pol and RS-2 fine quad pol wide scene.

In order to demonstrate the stability of the classifier we show the classified examples of detected oil spills and look alikes on both RS- 2 and and TS-X imagery. The matrices in Table 2 and 3 display the outcome of applying 10 classifiers (trained with different random initial weights) on reference samples of all four classes. Each column contains the percentages of samples from one reference class that, each column adds up to $100 \%$ and the diagonal entries of the Tables indicate correctly classified samples. The percentage values did not differ more than $3.7 \%$ from the computed mean for each individual classifier. This indicates a high stability of our neural network approach with respect to the training process.

Table 2. Classification results for reference data samples from each class, averaged over different neural network topologies, RS-2 dataset. Columns add up to $100 \%$.

\begin{tabular}{|c||c|c|c|c|}
\hline \multicolumn{1}{|c||}{} & \multicolumn{4}{c|}{ Reference Class } \\
\hline \hline ANN classification & CO & EM & LA & OW \\
\hline CO & $100.0 \%$ & $\%$ & $0 \%$ & $0 \%$ \\
\hline EM & $0 \%$ & $96.2 \%$ & $0 \%$ & $0 \%$ \\
\hline LA & $0 \%$ & $0 \%$ & $99.0 \%$ & $2.7 \%$ \\
\hline OW & $0 \%$ & $3.8 \%$ & $1.0 \%$ & $97.3 \%$ \\
\hline
\end{tabular}

Table 3. Classification results for reference data samples from each class, averaged over different neural network topologies, TS-X dataset . Columns add up to $100 \%$.

\begin{tabular}{|c||c|c|c|c|}
\hline \multicolumn{1}{|c||}{} & \multicolumn{4}{c|}{ Reference Class } \\
\hline \hline ANN classification & CO & EM & LA & OW \\
\hline CO & $100.0 \%$ & $\%$ & $0 \%$ & $0 \%$ \\
\hline EM & $0 \%$ & $95.4 \%$ & $0 \%$ & $0 \%$ \\
\hline LA & $0 \%$ & $0 \%$ & $99.3 \%$ & $3.8 \%$ \\
\hline OW & $0 \%$ & $4.6 \%$ & $0.7 \%$ & $96.2 \%$ \\
\hline
\end{tabular}

\section{CONCLUSIONS}

A combination of different polarimetric features have exploited to characterize dark spots on near coincident $\mathrm{C}$ and $\mathrm{X}$-band quad-polarisation SAR data using Artificial Neural Network as a classifier. Classification accuracy assessment shows the robustness of the proposed methodology to correctly identify different types of oil spills and look-alikes from the validation dataset. A first analysis of redundancy among these features indicates the directions for further investigation on selection of optimal features combinations which can help to avoid computational and memory overhead. These features clearly deserve attention in future studies. Additionally, the Proposed methodology is tuned for operational Near Real Time Services with average processing time of 10-12 $\mathrm{min}$ for a standard TS-X Quad-Pol StripMAP scene and 7-8 min for RS-2 Fine Quad-Pol scene.

\section{REFERENCES}

[1] D. Velotto, F. Nunziata, M. Migliaccio, and S. Lehner, "Dual-Polarized TerraSAR-X Data for Oil-Spill Observation," Geoscience and Remote Sensing, IEEE Transactions on, vol. 49, no. 12, pp. 1114-1118, 2011.

[2] M. Migliaccio, A. Gambardella, and M. Tranfaglia, "SAR polarimetry to observe oil spills," Geoscience and Remote Sensing, IEEE Transactions on, vol. 45, no. 2, pp. 506-511, 2007.

[3] M. Migliaccio, F. Nunziata, and A. Gambardella, "On the co-polarized phase difference for oil spill observation," International Journal of Remote Sensing, vol. 30, pp. 1587-1602, 2009.

[4] S. Skrunes, C. Brekke, T. Eltoft, and V. Kudryavtsev, "Comparing Near-Coincident C-and X-Band SAR Acquisitions of Marine Oil Spills," Geoscience and Remote Sensing, IEEE Transactions on, vol. 53, no. 9, pp. 19581975, 2014.

[5] S. Skrunes, C. Brekke, and T. Eltoft, "Characterization of marine surface slicks by Radarsat-2 multipolarization features," Geoscience and Remote Sensing, IEEE Transactions on, vol. 52, no. 9, pp. 5302-5319, 2014.

[6] A. H. S. Solberg, "Remote sensing of ocean oil-spill pollution," Proceedings of the IEEE, vol. 100, no. 10, pp. 2931-2945, 2012.

[7] J. Mittermayer and H. Runge, "Conceptual studies for exploiting the terrasar-x dual receive antenna," in Geoscience and Remote Sensing Symposium, 2003. IGARSS '03. Proceedings. 2003 IEEE International, vol. 3, July 2003, pp. 2140-2142.

[8] H. Breit, T. Fritz, U. Balss, M. Lachaise, A. Niedermeier, and M. Vonavka, "Terrasar-x sar processing and products," Geoscience and Remote Sensing, IEEE Transactions on, vol. 48, no. 2, pp. 727-740, Feb 2010.

[9] B. Zhang, W. Perrie, X. Li, and W. G. Pichel, "Mapping sea surface oil slicks using radarsat-2 quad-polarization sar image," Geophysical Research Letters, vol. 38, no. 10, pp. 1-5, 2011, 110602. [Online]. Available: http://dx.doi.org/10.1029/2011GL047013

[10] J. Praks, E. Koeniguer, and M. Hallikainen, "Alternatives to Target Entropy and Alpha Angle in SAR Polarimetry," Geoscience and Remote Sensing, IEEE Transactions on, vol. 47, no. 7, pp. 2262-2274, July 2009.

[11] P. Liu, X. Li, J. J. Qu, W. Wang, C. Zhao, and W. Pichel, "Oil spill detection with fully polarimetric UAVSAR data," Marine Pollution Bulletin, vol. 62, no. 12, pp. 2611-2618, 2011. 